\title{
On 2D Viscoelasticity with Small Strain
}

\author{
Zhen Lei *
}

\begin{abstract}
An exact two-dimensional rotation-strain model describing the motion of Hookean incompressible viscoelastic materials is constructed by the polar decomposition of the deformation tensor. The global existence of classical solutions is proved under the smallness assumptions only on the size of initial strain tensor. The proof of global existence utilizes the weak dissipative mechanism of motion, which is revealed by passing the partial dissipation to the whole system.
\end{abstract}

Keyword: Rotation-strain model, viscoelastic fluids, partial dissipation, complex fluids.

\section{Introduction}

Viscoelastic materials include a wide range of fluids with elastic properties, as well as solids with fluid properties. Let us start with the standard description of general mechanical evolutions to introduce some notations and definitions. Any deformation can be represented by a flow map (particle trajectory) $x(t, X), 0 \leq t<T$, a timedependent family of orientation-preserving diffeomorphisms. $X$ is the original labeling (Lagrangian coordinate) of the particles. $X$ is also referred to as the material/reference coordinate. $x$ is then the observer's (Eulerian) coordinate. In general, the velocity field $u(t, x)$ is defined as the time derivative of the flow map, which is a vector field defined on the Eulerian coordinate:

$$
u(t, x)=x_{t}(t, X(t, x))
$$

The deformation tensor $\widetilde{F}$ in Lagrangian coordinate is defined by

$$
\widetilde{F}_{i j}(t, X)=\frac{\partial x_{i}}{\partial X_{j}}(t, X) .
$$

When one uses the Eulerian description, the deformation gradient tensor $F$ in spatial coordinate is defined by

$$
F(t, x)=\widetilde{F}(t, X(t, x)) .
$$

\footnotetext{
${ }^{*}$ School of Mathematical Sciences, Fudan University, Shanghai 200433, P. R. China. email: leizhn@yahoo.com,zlei@fudan.edu.cn
} 
Recently, an extensively studied system modelling the motion of isotropic incompressible viscoelastic materials of Oldroyd-B type takes:

$$
\left\{\begin{array}{l}
\nabla \cdot u=0 \\
u_{t}+u \cdot \nabla u+\nabla p=\mu \Delta u+\nabla \cdot\left(\frac{\partial W(F)}{\partial F} F^{T}\right), \\
F_{t}+u \cdot \nabla F=\nabla u F
\end{array}\right.
$$

where $p(t, x)$ is the pressure, $\mu$ is the viscosity and $W(F)$ is the elastic energy function. The global existence of classical solutions near equilibrium to (1.1) in the 2D Hookean case was established by Lin, Liu and Zhang [17] (see also Lei and Zhou [15] via the incompressible limit method). Later on, Lei, Liu and Zhou [14] proved the global existence of classical small solutions to system (1.1) in both 2D and 3D cases (see also Chen and Zhang [3]). Very recently, an improved result was obtained by Kessenich [9] by removing the dependence of the smallness of the initial data on the viscosity via the hyperbolic energy method.

To study the different contributions of strain and rotation parts, Friedrich [5] observed that the smallness of the strain in nonlinear elasticity can be realized through the polar decomposition of the deformation tensor. John [6, 7] showed that no pointwise estimate for rotations in terms of strains can exist even in the case of small strain. In the work of Friedrich and John, no PDE is involved. From the PDE point of view, Liu and Walkington [19] considered approximating systems resulting from the special linearization of the original system with respect to the strain. Lei, Liu and Zhou [13] constructed a 2D rotation-strain viscoelastic model and proved the global existence their classical solutions with small strain. One shortcoming of the model in [13] is that the dynamics of strain and rotation are not equivalent to that of the deformation tensor.

One main concern of this paper is to construct an exact rotation-strain model for motions of Hookean viscoelastic materials of Oldroyd-B type, which takes

$$
\left\{\begin{array}{l}
\nabla \cdot u=0 \\
u_{t}+u \cdot \nabla u+\nabla p=\mu \Delta u+\nabla \cdot\left(V V^{T}\right)+2 \nabla \cdot V, \\
V_{t}+u \cdot \nabla V=D(u)+\frac{1}{2}\left(\nabla u V+V \nabla u^{T}\right) \\
\quad+\frac{1}{2} \omega_{12}(u)(V A-A V)-\frac{1}{2} \gamma(V A-A V), \\
\nabla^{\perp} \theta_{t}+u \cdot \nabla \nabla^{\perp} \theta=\frac{1}{2} \Delta u-\left(\begin{array}{c}
-\nabla_{2} u \cdot \nabla \theta \\
\nabla_{1} u \cdot \nabla \theta
\end{array}\right)+\nabla^{\perp} \gamma .
\end{array}\right.
$$

Here $V$ is the strain part of the deformation tensor, $\theta$ is the rotation angle and $\gamma$ is a nonlinear term. The derivation of this model will be presented in section 2 . It seems that the velocity field $u$ is the only dissipative unknown. We will prove the global existence of classical solutions to (1.2) for small initial strain $V$ and initial gradient of $\theta$ ( $\theta$ may be arbitrarily large). The proof utilizes the weak dissipative mechanism of 
system (1.2) by passing the dissipation of the velocity field $u$ to the strain tensor $V$ and the gradient of the angle variable $\nabla \theta$. Note that the equations for $u$ and $V$ have already formed an closed dynamical system. However, our proof needs the use of the equation for $\theta$ to carry out the weak dissipative mechanism of the whole system. Without the help of the equation for $\theta$ and the underlying physical relationship between $V$ and $\theta$ (see Lemma 3.3), the corresponding analysis issue is still open.

At last, let us also cite some related results on elastodynamics when the viscosity $\mu$ is zero. The global existence of 3D compressible elastodynamics was established by Agemi [1] and Sideris [20] independently under the null condition and small initial data. An earlier almost global existence result can be founded in John and Klainerman [8] (see also Klainerman and Sideris [11]). Hyperbolic energy method involving Klainerman's vector field developed by Klainerman [10] (see also Christodoulou [4]) plays an essential role in [11, 20]. The incompressible case was later on studied by Sideris and Thomases in [21, 22, 23]. A natural question to ask is that what happens for the solutions of (1.1) when the viscosity vanishes, which is answered very recently by Kessenich [9] in the case of 3D. Other results on Oldroyd-B model can be found in [2, 12, 16, 18.

The remaining part of this paper is organized as follows. In section 2 we present the derivation of the rotation-strain model. Then the main result of this paper is stated. Section 3 is devoted to exploring some of the intrinsic properties of the viscoelastic system. The proof of global existence theorem is completed in section 4 .

\section{Derivation of the Strain-Rotation Model}

Constitutive relations generally involve the deformation tensor $F$. In particular, the polar decomposition of $F$ is important. For any non-singular matrix $F$, it is easy to see that $F F^{T}$ is positive definite symmetric. Thus, there exists a unique positive definite symmetric matrix $I+V$ such that $(I+V)^{2}=F F^{T}$, where $I$ is the $2 \times 2$ identity matrix. Then the matrix $R=(I+V)^{-1} F$ is orthogonal. Consequently the non-singular matrix $F$ can be decomposed uniquely in the form

$$
F=(I+V) R
$$

where $R$ is orthogonal

$$
R R^{T}=I
$$

and $I+V$ positive definite symmetric

$$
V=V^{T}
$$

Physically, this means that the deformation is decomposed into stretching and rotation. Following a suggestion by K. O. Friedrichs [5], the tensor $I+V$ is called the left stretch tensor, $V$ the strain matrix and $R$ the rotation matrix.

Our first goal is to formulate the viscoelastic system in terms of the velocity $u$ and the strain $V$. We first look at the momentum equation, the second equation in 
(1.1). We restrict our discussions below to a special elastic energy functional of the Hookean linear elasticity $W(F)=\frac{1}{2}|F|^{2}$, it does not reduce the essential difficulties for mathematical analysis. Indeed, all the results we describe here can easily be generalized to a more general class of elastic energy functionals of the deformation tensor $F$ (see [14]). In terms of the strain matrix $V$, the momentum equation takes the form of

$$
u_{t}+u \cdot \nabla u+\nabla p=\mu \Delta u+\nabla \cdot\left(V V^{T}\right)+2 \nabla \cdot V
$$

where we used the equation (2.1) and (2.2). We point out here that through this paper, $\nabla$ with no indices stands for the derivative with respect to the spatial coordinate $x$. For a matrix-valued function $A,(\nabla \cdot B)_{i}$ means $\nabla_{j} B_{i j}$, where summation over repeated indices will always be well understood.

In order to get a dynamical equation for $V$, we plug (2.1) into the third equation of system (1.1). Denote the material derivatives of a quantity $Q$ by

$$
\dot{Q}=Q_{t}+u \cdot \nabla Q
$$

We have

$$
\dot{V} R+(I+V) \dot{R}=\nabla u(I+V) R .
$$

Noting that $R$ is orthogonal, we deduce that

$$
\dot{V}+(I+V) \dot{R} R^{T}=\nabla u(I+V) .
$$

By transposing the above equality (2.4), it is rather easy to see

$$
\dot{V}+R \dot{R}^{T}(I+V)=(I+V) \nabla u^{T} .
$$

Now let us write the orthogonal matrix $R$ as

$$
R=\left(\begin{array}{cc}
\cos \theta & -\sin \theta \\
\sin \theta & \cos \theta
\end{array}\right)
$$

It is immediately verified that

$$
\dot{R} R^{T}=\left(\begin{array}{cc}
-\sin \theta & -\cos \theta \\
\cos \theta & -\sin \theta
\end{array}\right)\left(\begin{array}{cc}
\cos \theta & \sin \theta \\
-\sin \theta & \cos \theta
\end{array}\right) \dot{\theta}=\left(\begin{array}{cc}
0 & -1 \\
1 & 0
\end{array}\right) \dot{\theta}
$$

Define

$$
A=\left(\begin{array}{cc}
0 & -1 \\
1 & 0
\end{array}\right)
$$

we have

$$
\dot{R} R^{T}=A \dot{\theta}, \quad V A+A V=A \operatorname{tr} V \quad A^{T}=A^{-1}=-A .
$$

Thus, it follows from (2.4), (2.5) and (2.7) that

$$
(I+V) A \dot{\theta}-A^{T}(I+V) \dot{\theta}=\nabla u(I+V)-(I+V) \nabla u^{T}
$$


which is equivalent to

$$
\begin{aligned}
\dot{\theta} I & =-\frac{1}{2+\operatorname{tr} V} A\left[\nabla u(I+V)-(I+V) \nabla u^{T}\right] \\
& =-\frac{1}{2+\operatorname{tr} V}\left[2 \omega_{12}(u)+\nabla_{k} u_{1} V_{k 2}-\nabla_{k} u_{2} V_{k 1}\right] I \\
& =-\omega_{12}(u) I+\gamma I,
\end{aligned}
$$

where

$$
\omega(u)=\frac{1}{2}\left(\nabla u-\nabla u^{T}\right)
$$

is the virticity tensor and

$$
\gamma=\frac{1}{2+\operatorname{tr} V}\left[\operatorname{tr} V \omega_{12}(u)-\left(\nabla_{k} u_{1} V_{k 2}-\nabla_{k} u_{2} V_{k 1}\right)\right]
$$

Consequently, we obtain that

$$
\dot{\theta}=-\omega_{12}(u)+\gamma
$$

Next, add up (2.4) and (2.5) and then insert (2.7) and (2.10) into the resulting equation, we obtian

$$
\begin{aligned}
\dot{V}= & \frac{1}{2}\left[\nabla u(I+V)+(I+V) \nabla u^{T}\right] \\
& -\frac{1}{2}\left[(I+V) \dot{R} R^{T}+R \dot{R}^{T}(I+V)\right] \\
= & \frac{1}{2}\left(\nabla u+\nabla u^{T}\right)+\frac{1}{2}\left(\nabla u V+V \nabla u^{T}\right) \\
& +\frac{1}{2}\left[(I+V) A+A^{T}(I+V)\right] \omega_{12}(u) \\
& \quad-\frac{1}{2} \gamma\left[(I+V) A+A^{T}(I+V)\right] \\
= & \frac{1}{2}\left(\nabla u+\nabla u^{T}\right)+\frac{1}{2}\left(\nabla u V+V \nabla u^{T}\right) \\
& \quad+\frac{1}{2} \omega_{12}(u)(V A-A V)-\frac{1}{2} \gamma(V A-A V),
\end{aligned}
$$

where in the last equality we used (2.7). If we denote the symmetric part of the gradient of velocity by

$$
D(u)=\frac{1}{2}\left(\nabla u+\nabla u^{T}\right)
$$

we can rewrite the above equation (2.11) as

$$
\begin{aligned}
V_{t} & +u \cdot \nabla V=D(u)+\frac{1}{2}\left(\nabla u V+V \nabla u^{T}\right) \\
& +\frac{1}{2} \omega_{12}(u)(V A-A V)-\frac{1}{2} \gamma(V A-A V) .
\end{aligned}
$$


In order to formulate an incompressible dynamical problem, the equations of mass conservation $\nabla \cdot u=0$ and balance of momentum (2.3) supplemented by the transport equation (2.13) have in fact made up of a closure system. However, to prove the global existence, we must include an equation for $\nabla \theta$ to carry out some intrinsic properties of the rotation-strain viscoelastic system, which will be seen more clear in section 4 .

Let

$$
\nabla^{\perp}=\left(\begin{array}{c}
-\nabla_{2} \\
\nabla_{1}
\end{array}\right)
$$

and then apply $\nabla^{\perp}$ to (2.10) to yield

$$
\nabla^{\perp} \theta_{t}+u \cdot \nabla \nabla^{\perp} \theta=\frac{1}{2} \Delta u-\left(\begin{array}{c}
-\nabla_{2} u \cdot \nabla \theta \\
\nabla_{1} u \cdot \nabla \theta
\end{array}\right)+\nabla^{\perp} \gamma
$$

This equation will be used to explore the weak dissipation of $\nabla \theta$, which eventually shows that $V$ is also weakly dissipative. The equations (2.3), (2.13) and (2.15) form the rotation-strain model (1.2) for viscoelastic fluids. For future use, we also need an equation of $\nabla \theta$. For this purpose, we apply the gradient operator $\nabla$ to (2.10) to get

$$
\nabla \theta_{t}+u \cdot \nabla \nabla \theta=-\left(\begin{array}{c}
\nabla_{1} u \cdot \nabla \theta \\
\nabla_{2} u \cdot \nabla \theta
\end{array}\right)-\nabla \omega_{12}(u)+\nabla \gamma
$$

We will consider the Cauchy problem or the periodic boundary-value problem of system (1.2). The initial data takes of the form

$$
u(0, x)=u_{0}(x), \quad V(0, x)=V_{0}(x), \quad \nabla \theta(0, x)=\nabla \theta_{0}(x), \quad x \in \Omega .
$$

where $\Omega \subseteq R^{2}$ is two dimensional torus or the entire space. The above initial data will be imposed on the following constraints

$$
\left\{\begin{array}{l}
\nabla \cdot u_{0}=0 \\
\operatorname{det}\left(I+V_{0}\right)=1, \\
\nabla \cdot V_{0}=A\left(I+V_{0}\right) \nabla \theta_{0} .
\end{array}\right.
$$

The first two are just the consequences of the incompressibility and the last one is understood as the consistency condition for changing of variables (see Lemma 3.1, Lemma 3.3, Remark 3.2 and Remark 3.4 in the next section).

Remark 2.1. In our earlier work [13], the rotation matrix $R$ satisfies a spontaneous but specified transport equation:

$$
R_{t}+u \cdot \nabla R=-A R \omega_{12}(u)=\omega(u) R
$$

Consequently, it follows that

$$
\theta_{t}+u \cdot \nabla \theta=-\omega_{12}
$$


This combining the third equation of system (1.1) gives a non-symmetric transport equation for $V$. While in the model (1.2), noting (2.7) and with the aid of (2.10), it is rather easy to find that

$$
R_{t}+u \cdot \nabla R=A R \dot{\theta}=-A R \omega_{12}(u)+\gamma A R .
$$

The last term of the right side of the above equation or the one $\gamma$ in (2.10) represents corrections of our previous model, which leads to that the strain matrix must be symmetric. However, the corrections reflect the intrinsic properties of motions of viscoelastic fluids and the underlying physical origins.

For the rotation-strain model (1.2), we will prove the following theorem:

Theorem 2.2. Consider the Cauchy problem or the periodic initial-boundary value problem for the rotation-strain viscoelastic model (1.2) and (2.17) with the intrinsic physical constraints (2.18) on the initial data. Then there exists a unique global classical solution $(u, V, \nabla \theta)$ which satisfies

$$
\begin{aligned}
& \|u\|_{H^{2}(\Omega)}^{2}+\|V\|_{H^{2}(\Omega)}^{2}+\left\|\Delta u+\frac{1}{\mu} \nabla^{\perp} \theta\right\|^{2}+\mu \int_{0}^{\infty}\left[\|\nabla u\|_{H^{2}}^{2}\right. \\
& \left.+\left\|\nabla\left(\Delta u+\frac{1}{\mu} \nabla^{\perp} \theta\right)\right\|^{2}+\left\|\nabla\left(\Delta u+\frac{1}{\mu} \nabla \cdot V\right)\right\|^{2}\right] d t \leq \frac{\mu^{4}\left(1+\mu^{4}\right)}{C^{2}\left(1+\mu^{10}\right)},
\end{aligned}
$$

if the initial data $u_{0}, V_{0} \in H^{2}(\Omega)$ and $\nabla \theta_{0} \in H^{1}(\Omega)$ and

$$
\left\|u_{0}\right\|_{H^{2}(\Omega)}^{2}+\left\|V_{0}\right\|_{H^{2}(\Omega)}^{2}+\left\|\nabla \theta_{0}\right\|_{H^{1}(\Omega)}^{2}<\frac{\mu^{8}}{M\left(1+\mu^{10}\right)},
$$

where $\Omega \subseteq R^{2}$ is two dimensional torus or the entire space, $C$ and $M\left(M>2 C^{3}\right)$ are big enough constants independent of $t$ and $\mu$.

The proof of the above theorem relies on a local existence theorem and a priori energy estimates. The proof of local existence is standard and can be similarly done as in [14, 16], thus is omitted here. Below we will only present the a priori estimate in Theorem 2.2 .

Remark 2.3. In the case of $\Omega=\mathrm{T}^{2}$ being a two-dimensional torus, we in fact recovered the results in our earlier work [14. To see this, we set

$$
\bar{\theta}_{0}=\frac{1}{\left|\mathrm{~T}^{2}\right|} \int_{\mathrm{T}^{2}} \theta_{0} d x
$$

By Poincaré's inequality, we have

$$
\left\|\theta_{0}-\bar{\theta}_{0}\right\|_{L^{\infty}} \leq C\left\|\theta_{0}-\bar{\theta}_{0}\right\|_{H^{2}} \leq C\left\|\nabla \theta_{0}\right\|_{H^{1}}
$$


Thus, if we define

$$
R_{0}=\left(\begin{array}{cc}
\cos \bar{\theta}_{0} & -\sin \bar{\theta}_{0} \\
\sin \bar{\theta}_{0} & \cos \bar{\theta}_{0}
\end{array}\right)
$$

then

$$
F(0, x)=[(I+V) R]_{t=0}=R_{0}+E_{0}
$$

where

$$
E_{0}=\left.\left[R-R_{0}+V R\right]\right|_{t=0}
$$

is a small disturbance near a constant equilibrium $R_{0}$ by $(2.20)$, since $\left\|\nabla \theta_{0}\right\|_{H^{1}}$ is assumed to be small in Theorem 2.2. In [14], we studied the general viscoelastic model including an equation for the deformation tensor $F$ and proved the global existence of classical solutions near constant equilibrium in both two and three-dimensional spaces. However, our results and proofs here are still of importance even in the periodic case because they provide us a better understanding of the physical background of the system.

\section{Special Structures of the System}

In this section, we will explore some of the intrinsic properties of the viscoelastic system mentioned in section 2. These properties reflect the underlying physical origins of the problem and in the meantime, and are also essential to the proof of the global existence result here.

The following lemma will be used to explore the weak dissipation of $V$.

Lemma 3.1. Assume that the second equality of (2.18) is satisfied and $(u, V)$ is the solution of system (1.2). Then the following is always true:

$$
\operatorname{det}(I+V)=1
$$

for all the latter time $t \geq 0$. In other words, we have

$$
\operatorname{tr} V=-\operatorname{det} V
$$

Proof. Using the identity that for any non-singular matrix $F$,

$$
\frac{\partial \operatorname{det} F}{\partial F}=\operatorname{det} F F^{-T} \text {, }
$$

we have

$$
\begin{aligned}
& (\operatorname{det}(I+V))_{t}+u \cdot \nabla(\operatorname{det}(I+V)) \\
& =\operatorname{det}(I+V)(I+V)_{j i}^{-1} \dot{V}_{i j} \\
& =\operatorname{tr}\left\{\dot{V}+\left(\begin{array}{cc}
V_{22} & -V_{12} \\
-V_{12} & V_{11}
\end{array}\right) \dot{V}\right\} .
\end{aligned}
$$


Return to (2.11), we in fact have that

$$
\dot{V}=D(u)+\frac{1}{2}\left(\nabla u V+V \nabla u^{T}\right)-\frac{1}{2}(V A-A V) \dot{\theta} .
$$

By (3.3), (3.4), we arrive at

$$
\begin{aligned}
& (\operatorname{det}(I+V))_{t}+u \cdot \nabla(\operatorname{det}(I+V)) \\
& =\operatorname{tr} D(u)-\frac{1}{2} \operatorname{tr}(V A-A V) \dot{\theta}+\operatorname{tr}\left\{\nabla u V+\left(\begin{array}{cc}
V_{22} & -V_{12} \\
-V_{12} & V_{11}
\end{array}\right) D(u)\right\} \\
& +\operatorname{tr}\left\{\left(\begin{array}{cc}
V_{22} & -V_{12} \\
-V_{12} & V_{11}
\end{array}\right) \nabla u V\right\}+\operatorname{tr}\left\{\left(\begin{array}{cc}
V_{22} & -V_{12} \\
-V_{12} & V_{11}
\end{array}\right)(V A-A V)\right\} .
\end{aligned}
$$

We will compute the right side of the above equality term by term. First of all, with the use of the first equation of (1.2), it is rather easy to see that

$$
\operatorname{tr} D(u)=0 .
$$

Making use of (2.6), we deduce that

$$
\operatorname{tr}(V A-A V)=\operatorname{tr}\left(\begin{array}{cc}
2 V_{12} & V_{22}-V_{11} \\
V_{22}-V_{11} & -2 V_{12}
\end{array}\right)=0 .
$$

According to the expression of the Cauchy strain tensor (2.12), the third term of (3.5) can be calculated as

$$
\begin{aligned}
& \operatorname{tr}\left\{\nabla u V+\left(\begin{array}{cc}
V_{22} & -V_{12} \\
-V_{12} & V_{11}
\end{array}\right) D(u)\right\} \\
& =\nabla_{1} u_{1} V_{11}+\nabla_{2} u_{1} V_{12}+\nabla_{1} u_{2} V_{12}+\nabla_{2} u_{2} V_{22} \\
& \quad+V_{22} \nabla_{1} u_{1}-V_{12}\left(\nabla_{1} u_{2}+\nabla_{2} u_{1}\right)+V_{11} \nabla_{2} u_{2} \\
& =\operatorname{tr} V \nabla \cdot u=0 .
\end{aligned}
$$

Similarly, by a straightforward computation, we find that

$$
\begin{aligned}
& \operatorname{tr}\left\{\left(\begin{array}{cc}
V_{22} & -V_{12} \\
-V_{12} & V_{11}
\end{array}\right) \nabla u V\right\} \\
&=\operatorname{tr}\left\{\left(\begin{array}{cc}
V_{22} & -V_{12} \\
-V_{12} & V_{11}
\end{array}\right)\left(\begin{array}{cc}
\nabla_{1} u_{1} V_{11}+\nabla_{2} u_{1} V_{12} & \nabla_{1} u_{1} V_{12}+\nabla_{2} u_{1} V_{22} \\
\nabla_{1} u_{2} V_{11}+\nabla_{2} u_{2} V_{12} & \nabla_{1} u_{2} V_{12}+\nabla_{2} u_{2} V_{22}
\end{array}\right)\right\} \\
&=V_{22}\left(\nabla_{1} u_{1} V_{11}+\nabla_{2} u_{1} V_{12}\right)+V_{11}\left(\nabla_{1} u_{2} V_{12}+\nabla_{2} u_{2} V_{22}\right) \\
&-V_{12}\left[\left(\nabla_{1} u_{2} V_{11}+\nabla_{2} u_{2} V_{12}\right)+\left(\nabla_{1} u_{1} V_{12}+\nabla_{2} u_{1} V_{22}\right)\right] \\
&= V_{11} V_{22} \nabla \cdot u+V_{12}\left(V_{22} \nabla_{2} u_{1}+V_{11} \nabla_{1} u_{2}\right) \\
&-V_{12}\left[\nabla_{1} u_{2} V_{11}+\nabla_{2} u_{1} V_{22}+V_{12} \nabla \cdot u\right] \\
&= 0 .
\end{aligned}
$$


It remains to deal with the last term of (3.5). Recall (2.6) once more, it follows from a simple calculation that

$$
\begin{aligned}
& \operatorname{tr}\left\{\left(\begin{array}{cc}
V_{22} & -V_{12} \\
-V_{12} & V_{11}
\end{array}\right)(V A-A V)\right\} \\
& =\operatorname{tr}\left\{\left(\begin{array}{cc}
V_{22} & -V_{12} \\
-V_{12} & V_{11}
\end{array}\right)\left(\begin{array}{cc}
2 V_{12} & V_{22}-V_{11} \\
V_{22}-V_{11} & -2 V_{12}
\end{array}\right)\right\} \\
& =2 V_{22} V_{12}-2 V_{12}\left(V_{22}-V_{11}\right)-2 V_{11} V_{12}=0 .
\end{aligned}
$$

Finally, combining the above equalities (3.5) with (3.6) $-(3.10)$, we arrive at

$$
(\operatorname{det}(I+V))_{t}+u \cdot \nabla(\operatorname{det}(I+V))=0 .
$$

This completes the proof Lemma 3.1.

Remark 3.2. The incompressibility can be exactly represented as

$$
\operatorname{det} F=1 \text {. }
$$

The usual incompressible condition $\nabla \cdot u=0$, the first equation in (1.2), is the direct consequence of this identity. On the other hand, $\operatorname{det}(I+V)=1$ is also a direct conclusion of (3.12) since $R$ is an orthogonal matrix. However, the above Lemma illustrates the incompressible consistency of the the system (1.2).

To show the global existence of the classical small strain solutions to system (1.2), we still need to show that the pressure is a high order term, which will be seen more clear in the next section. To do so, we will use the following lemma to find out the weak dissipation of $\nabla \theta$, which will play an important role for our proof below.

Lemma 3.3. Assume that the third equality of (2.18) is satisfied. Then any solution $(u, V)$ of the system (1.2)-(2.17) will satisfy the following identity

$$
\nabla \cdot V=A(I+V) \nabla \theta
$$

for all the latter time $t \geq 0$.

Proof. We rewrite the third equation of (1.2) as

$$
V_{t}+u \cdot \nabla V=D(u)+\frac{1}{2}\left(\nabla u V+V \nabla u^{T}\right)-\frac{1}{2}(V A-A V) \dot{\theta},
$$

and then apply $\nabla \cdot$ to the resulting equation (3.14) to yield

$$
\begin{aligned}
& \nabla \cdot V_{t}+u \cdot \nabla \nabla \cdot V \\
&=\frac{1}{2} \Delta u+\frac{1}{2} A V \nabla \dot{\theta}-\left(\begin{array}{c}
\nabla_{j} u \cdot \nabla V_{1 j} \\
\nabla_{j} u \cdot \nabla V_{2 j}
\end{array}\right)+\frac{1}{2} \nabla \cdot\left(\nabla u V+V \nabla u^{T}\right) \\
&-\frac{1}{2} V A \nabla \dot{\theta}-\frac{1}{2} \nabla \cdot(V A-A V) \dot{\theta} \\
&= \frac{1}{2} \Delta u+\frac{1}{2} A V \nabla \dot{\theta}-\left(\begin{array}{c}
\nabla_{j} u \cdot \nabla V_{1 j} \\
\nabla_{j} u \cdot \nabla V_{2 j}
\end{array}\right)+\frac{1}{2} \nabla \cdot\left(\nabla u V+V \nabla u^{T}\right) \\
&-\frac{1}{2} V A \nabla \dot{\theta}-\frac{1}{2} \nabla \cdot(V A+A V) \dot{\theta}+A \nabla \cdot V \dot{\theta} .
\end{aligned}
$$


By the first equation of (1.2) and a straightforward computation, we obtain

$$
-\left(\begin{array}{c}
\nabla_{j} u \cdot \nabla V_{1 j} \\
\nabla_{j} u \cdot \nabla V_{2 j}
\end{array}\right)+\frac{1}{2} \nabla \cdot\left(\nabla u V+V \nabla u^{T}\right)=\frac{1}{2} \nabla \cdot\left(\nabla u V-V \nabla u^{T}\right) .
$$

In view of (2.10), we can rewrite the last term of (3.15) as

$$
A \nabla \cdot V \dot{\theta}=[\omega(u)+\gamma A] \nabla \cdot V .
$$

Thus, it follows from (3.15), (3.16) and (3.17) that

$$
\begin{aligned}
& \nabla \cdot V_{t}+u \cdot \nabla \nabla \cdot V \\
& =\frac{1}{2} \Delta u+\frac{1}{2} A V \nabla \dot{\theta}+[\omega(u)+\gamma A] \nabla \cdot V-\frac{1}{2} V A \nabla \dot{\theta} \\
& \quad+\frac{1}{2} \nabla \cdot\left(\nabla u V-V \nabla u^{T}\right)-\frac{1}{2} \nabla \cdot(V A+A V) \dot{\theta} .
\end{aligned}
$$

On the other hand, combining (2.16) with (3.14), and splitting some terms of the resulting expression, we conclude that

$$
\begin{aligned}
\partial_{t} & {[A(I+V) \nabla \theta]+u \cdot \nabla[A(I+V) \nabla \theta] } \\
= & A D(u) \nabla \theta+\frac{1}{2} A\left(\nabla u V+V \nabla u^{T}\right) \nabla \theta-\frac{1}{2}(A V A+V) \dot{\theta} \nabla \theta \\
& +A(I+V) \nabla \dot{\theta}-A(I+V)\left(\begin{array}{c}
\nabla_{1} u \cdot \nabla \theta \\
\nabla_{2} u \cdot \nabla \theta
\end{array}\right) \\
= & A \nabla \dot{\theta}+\frac{1}{2} A V \nabla \dot{\theta}+\frac{1}{2} A V \nabla \dot{\theta}+A D(u) \nabla \theta+\frac{1}{2} A\left(\nabla u V+V \nabla u^{T}\right) \nabla \theta \\
& -\frac{1}{2}(A V A+V) \dot{\theta} \nabla \theta-A\left(\begin{array}{c}
\nabla_{1} u \cdot \nabla \theta \\
\nabla_{2} u \cdot \nabla \theta
\end{array}\right)-A V\left(\begin{array}{c}
\nabla_{1} u \cdot \nabla \theta \\
\nabla_{2} u \cdot \nabla \theta
\end{array}\right) .
\end{aligned}
$$

In view of (2.10) and the first equation of system (1.2), we obtain, after a straightforward calculation,

$$
A \nabla \dot{\theta}=\frac{1}{2} \Delta u+A \nabla \gamma .
$$

It is rather easy to see that

$$
A D(u) \nabla \theta-A\left(\begin{array}{c}
\nabla_{1} u \cdot \nabla \theta \\
\nabla_{2} u \cdot \nabla \theta
\end{array}\right)=\omega(u) A \nabla \theta
$$

and

$$
\frac{1}{2} A\left(\nabla u V+V \nabla u^{T}\right) \nabla \theta-A V\left(\begin{array}{c}
\nabla_{1} u \cdot \nabla \theta \\
\nabla_{2} u \cdot \nabla \theta
\end{array}\right)=\frac{1}{2} A\left(\nabla u V-V \nabla u^{T}\right) \nabla \theta .
$$

Using (2.7) and (2.10), we find that

$$
\begin{aligned}
& -\frac{1}{2}(A V A+V) \dot{\theta} \nabla \theta \\
& =-\frac{1}{2} A(V A+A V) \dot{\theta} \nabla \theta-\left(-\omega_{12}(u)+\gamma\right) V \nabla \theta \\
& =-\frac{1}{2} A(V A+A V) \dot{\theta} \nabla \theta+A \omega(u) V \nabla \theta-\gamma V \nabla \theta .
\end{aligned}
$$


Combining (3.19)-(3.23), we have

$$
\begin{aligned}
& \partial_{t}[A(I+V) \nabla \theta]+u \cdot \nabla[A(I+V) \nabla \theta] \\
& =\frac{1}{2} \Delta u+\frac{1}{2} A V \nabla \dot{\theta}+\omega(u) A(I+V) \nabla \theta-\frac{1}{2} A(V A+A V) \dot{\theta} \nabla \theta \\
& \quad+A \nabla \gamma+\frac{1}{2} A\left(\nabla u V-V \nabla u^{T}\right) \nabla \theta+\frac{1}{2} A V \nabla \dot{\theta}-\gamma V \nabla \theta
\end{aligned}
$$

Now, we subtract (3.24) from (3.18), and rearrange the right side terms of resulting equation. The outcome of this straightforward calculations is

$$
\begin{aligned}
\partial_{t} & {[\nabla \cdot V-A(I+V) \nabla \theta]+u \cdot \nabla[\nabla \cdot V-A(I+V) \nabla \theta] } \\
= & \omega(u)[\nabla \cdot V-A(I+V) \nabla \theta]-\frac{1}{2}(V A+A V) \nabla \dot{\theta}-\frac{1}{2} \nabla \cdot(V A+A V) \dot{\theta} \\
& +\frac{1}{2} \nabla \cdot\left(\nabla u V-V \nabla u^{T}\right)-A \nabla \gamma+\gamma A \nabla \cdot V \\
& +\left[\frac{1}{2} A(V A+A V) \dot{\theta}-\frac{1}{2} A\left(\nabla u V-V \nabla u^{T}\right)+\gamma V\right] \nabla \theta \\
= & \omega(u)[\nabla \cdot V-A(I+V) \nabla \theta]-\frac{1}{2} \nabla \cdot[(V A+A V) \dot{\theta}] \\
& +\frac{1}{2} \nabla \cdot\left(\nabla u V-V \nabla u^{T}\right)-A \nabla \gamma+\gamma A \nabla \cdot V \\
& +\left[\frac{1}{2} A(V A+A V) \dot{\theta}-\frac{1}{2} A\left(\nabla u V-V \nabla u^{T}\right)+\gamma V\right] \nabla \theta .
\end{aligned}
$$

Our objective is to show that the expression $\nabla \cdot V-A(I+V) \nabla \theta$ satisfies some differential equation whose solution can be uniquely determined by the initial data. Recall (2.7) and (2.10), we get

$$
\begin{aligned}
- & \frac{1}{2} \nabla \cdot[(V A+A V) \dot{\theta}]+\frac{1}{2} \nabla \cdot\left(\nabla u V-V \nabla u^{T}\right)-A \nabla \gamma+\gamma A \nabla \cdot V \\
= & -\frac{1}{2} \nabla \cdot\left[\operatorname{tr} V A\left(-\omega_{12}(u)+\gamma\right)\right]-A \nabla \gamma+\gamma A \nabla \cdot V \\
& +\frac{1}{2} \nabla \cdot\left(\nabla u V-V \nabla u^{T}\right) \\
= & \left.\frac{1}{2} A \nabla\left[\operatorname{tr} V \omega_{12}(u)\right]-\frac{1}{2} A \nabla[(\operatorname{tr} V+2) \gamma)\right]+\gamma A \nabla \cdot V \\
& -\frac{1}{2} \nabla \cdot\left[A\left(\nabla_{k} u_{1} V_{k 2} \quad-\nabla_{k} u_{2} V_{k 1}\right)\right] \\
= & \frac{1}{2} A \nabla\left[\operatorname{tr} V \omega_{12}(u)-\left(\nabla_{k} u_{1} V_{k 2}-\nabla_{k} u_{2} V_{k 1}\right)\right]+\gamma A \nabla \cdot V \\
& \left.-\frac{1}{2} A \nabla[(\operatorname{tr} V+2) \gamma)\right] .
\end{aligned}
$$

Thus, by (2.9), we can easily deduce that

$$
\begin{aligned}
& -\frac{1}{2} \nabla \cdot[(V A+A V) \dot{\theta}]+\frac{1}{2} \nabla \cdot\left(\nabla u V-V \nabla u^{T}\right) \\
& -A \nabla \gamma+\gamma A \nabla \cdot V=\gamma A \nabla \cdot V .
\end{aligned}
$$


On the other hand, it follows from (2.7), (2.9) and (2.10) that

$$
\begin{aligned}
& \frac{1}{2} A(V A+A V) \dot{\theta}-\frac{1}{2} A\left(\nabla u V-V \nabla u^{T}\right)+\gamma V \\
= & -\gamma A A(I+V)-\gamma I-\frac{1}{2} \operatorname{tr} V\left[-\omega_{12}(u)\right. \\
& +\gamma] I-\frac{1}{2} A\left(\nabla u V-V \nabla u^{T}\right) \\
= & -\gamma A A(I+V)-\frac{1}{2}\left[-\operatorname{tr} V \omega_{12}(u)+\gamma(t r V+2)\right] I \\
& -\frac{1}{2} A\left(\nabla u V-V \nabla u^{T}\right) \\
= & -\gamma A A(I+V)-\frac{1}{2}\left(\nabla_{k} u_{1} V_{k 2}-\nabla_{k} u_{2} V_{k 1}\right) I \\
& +\frac{1}{2} A\left(\nabla u V+V \nabla u^{T}\right) \\
= & -\gamma A A(I+V) .
\end{aligned}
$$

Hence we conclude from (3.25)-(3.27) that

$$
\begin{aligned}
& \partial_{t}[\nabla \cdot V-A(I+V) \nabla \theta]+u \cdot \nabla[\nabla \cdot V-A(I+V) \nabla \theta] \\
& =[\omega(u)+\gamma A][\nabla \cdot V-A(I+V) \nabla \theta] .
\end{aligned}
$$

which concludes the proof of the lemma, since the above quantity will remain zero all the time with zero initial data.

Remark 3.4. In order to carry out the mechanical background of the above lemma, we again go back to the deformation tensor $F$. By its definition, the following identity

$$
\nabla \cdot F^{T}=0
$$

is proved in [14, 15]. Insert (2.1) into the above equality, we find

$$
\partial_{i} R_{i j}+R_{k j} \partial_{i} V_{i k}+V_{i k} \partial_{i} R_{k j}=0
$$

which implies

$$
\partial_{i} V_{i l}+R_{l j} \partial_{i} R_{i j}+R_{l j} V_{i k} \partial_{i} R_{k j}=0
$$

since $R$ is an orthogonal matrix. But then employing (2.2) a straightforward calculation yields (3.13).

\section{Proof of Theorem 2.2}

In this section we prove Theorem 2.2. Weak dissipations on the strain $V$ and the gradient of rotation angle $\nabla \theta$ are found by introducing auxiliary functions $w$ and $\Theta$ below. The way of defining such functions reveals the intrinsic dissipative nature of 
the system. In what follows, $\|\cdot\|$ is used to denote the standard $L^{2}\left(R^{2}\right)$ norm, $<\cdot, \cdot>$ and $(\cdot, \cdot)$ the $R^{d}$ and $L^{2}\left(R^{2}\right)^{d}$ inner products with $d=1,2$ or 4 . The proof is divided into four steps.

\section{Step 1. Standard Energy Estimates.}

By taking the $L^{2}$ inner product of the second and third equations of system (1.2) with $u$ and $V$, and then adding up the resulting equations together, we obtain

$$
\begin{aligned}
& \frac{1}{2} \frac{d}{d t} \int_{R^{2}}\left(|u|^{2}+|V|^{2}\right) d x+\mu\|\nabla u\|^{2} \\
= & -\int_{R^{2}} u \cdot \nabla \frac{|u|^{2}+|V|^{2}}{2}+<u, \nabla p>d x \\
& +\int_{R^{2}}<V, \frac{1}{2}\left(\nabla u V+V \nabla u^{T}\right)+\frac{1}{2} \omega_{12}(u)(V A-A V)>d x \\
& +\int_{R^{2}}<u, 2 \nabla \cdot V>+<V, D(u)>d x \\
& +\int_{R^{2}}<u, \nabla \cdot\left(V V^{T}\right)>d x-\int_{R^{2}}<V, \frac{1}{2} \gamma(V A-A V)>d x .
\end{aligned}
$$

On the other hand, the fourth equation of system (1.2) gives

$$
\begin{aligned}
& \frac{1}{2} \frac{d}{d t} \int_{R^{2}} 2 \operatorname{tr} V d x=-\int_{R^{2}} u \cdot \nabla \operatorname{tr} V d x+\int_{R^{2}} \operatorname{tr} D(u) d x \\
& \quad+\int_{R^{2}} \operatorname{tr}\left(\nabla u V+\frac{1}{2}(V A-A V)\left(\omega_{12}(u)-\gamma\right)\right) d x .
\end{aligned}
$$

Adding up the above two equation together, we have

$$
\begin{aligned}
& \frac{1}{2} \frac{d}{d t} \int_{R^{2}}\left(|u|^{2}+|V|^{2}+2 \operatorname{tr} V\right) d x+\mu\|\nabla u\|^{2} \\
& =-\int_{R^{2}} u \cdot \nabla\left(\frac{|u|^{2}+|V|^{2}}{2}+\operatorname{tr} V\right)+<u, \nabla p>d x+\int_{R^{2}} \operatorname{tr} D(u) d x \\
& \quad+\int_{R^{2}}<V, \frac{1}{2}\left(\nabla u V+V \nabla u^{T}\right)+\frac{1}{2} \omega_{12}(u)(V A-A V)>d x \\
& \quad+\int_{R^{2}}<u, 2 \nabla \cdot V>+<V, D(u)>d x+\int_{R^{2}} \operatorname{tr}(\nabla u V) d x \\
& \quad+\int_{R^{2}}<u, \nabla \cdot\left(V V^{T}\right)>d x-\int_{R^{2}}<V, \frac{1}{2} \gamma(V A-A V)>d x \\
& \quad+\int_{R^{2}} \operatorname{tr} \frac{1}{2}\left((V A-A V)\left(\omega_{12}(u)-\gamma\right)\right) d x .
\end{aligned}
$$

Noting that $V$ is symmetric, and with the aid of the first equation of (1.2), it is rather easy to see that

$$
\left\{\begin{array}{l}
-\int_{R^{2}} u \cdot \frac{1}{2}\left(|u|^{2}+|V|^{2}+2 \operatorname{tr} V\right)+<u, \nabla p>d x+\int_{R^{2}} \operatorname{tr} D(u) d x=0 \\
\int_{R^{2}}<u, 2 \nabla \cdot V>+<V, D(u)>d x+\int_{R^{2}} \operatorname{tr}(\nabla u V) d x=0
\end{array}\right.
$$


Noting the definition in (2.6) and (2.9), one has

$$
\begin{aligned}
& \int_{R^{2}}<V, \frac{1}{2}\left(\nabla u V+V \nabla u^{T}\right)+\frac{1}{2} \omega_{12}(u)(V A-A V)>d x \\
& \quad+\int_{R^{2}}<u, \nabla \cdot\left(V V^{T}\right)>d x-\int_{R^{2}}<V, \frac{1}{2} \gamma(V A-A V)>d x \\
& \quad+\int_{R^{2}} \operatorname{tr} \frac{1}{2}\left((V A-A V)\left(\omega_{12}(u)-\gamma\right)\right) d x \\
& =\int_{R^{2}}<V, \nabla u V>+<u, \nabla \cdot\left(V V^{T}\right)>d x \\
& \quad+\int_{R^{2}} \frac{1}{2}\left(\omega_{12}(u)-\gamma\right)(\operatorname{tr}(V A-A V)+<V, V A-A V>) d x \\
& =0 .
\end{aligned}
$$

Then, combining (4.1), (4.2) with (4.3), we have

$$
\frac{1}{2} \frac{d}{d t} \int_{R^{2}}\left(|u|^{2}+|V|^{2}+2 \operatorname{tr} V\right) d x+\mu\|\nabla u\|^{2}=0
$$

Finally, by using (3.2), we arrive at

$$
\frac{1}{2} \frac{d}{d t} \int_{R^{2}}\left(|u|^{2}+\left|V_{11}-V_{22}\right|^{2}+\left|V_{12}+V_{21}\right|^{2}\right) d x+\mu\|\nabla u\|^{2}=0 .
$$

By taking the $L^{2}$ inner product of the second and third equations of system (1.2) with $u$ and $2 V$, and then adding up the resulting equations together, we obtain

$$
\begin{aligned}
& \frac{1}{2} \frac{d}{d t} \int_{R^{2}}|u|^{2}+2|V|^{2} d x+\int_{R^{2}} u \cdot \nabla\left(\frac{|u|^{2}}{2}+|V|^{2}\right) d x+(u, \nabla p) \\
& =\left(V, \nabla u V+V \nabla u^{T}+\omega_{12}(u)(V A-A V)-\gamma(V A-A V)\right) \\
& \quad+\left(u, \nabla \cdot\left(V V^{T}\right)\right)+(u, 2 \nabla \cdot V)+(2 V, D(u))+\mu(u, \Delta u),
\end{aligned}
$$

which gives that

$$
\begin{aligned}
& \frac{1}{2} \frac{d}{d t} \int_{R^{2}}|u|^{2}+2|V|^{2} d x+\mu\|\nabla u\|^{2} \\
& \leq\|\nabla u\|_{L^{\infty}}\|V\|^{2} \\
& \leq C\|\nabla V\|\|\Delta V\|(\|\Delta \nabla u\|+\|\nabla u\|) \\
& \leq C \mu\|\nabla V\|_{L^{2}}\left(\|\nabla u\|^{2}+\|\Delta \nabla u\|^{2}+\frac{1}{\mu^{2}}\|\Delta V\|\right) .
\end{aligned}
$$

In order to exploit the higher order energy estimates, we apply $\Delta$ to the second and third equations of system (1.2), and then take $L^{2}$ inner product of the resulting 
equations with $\Delta u$ and $2 \Delta V$, respectively, to yield

$$
\begin{aligned}
& \frac{1}{2} \frac{d}{d t} \int_{R^{2}}|\Delta u|^{2}+2|\Delta V|^{2} d x+(\Delta u, \nabla \Delta p) \\
& \quad+\int_{R^{2}} u \cdot \nabla\left(\frac{|\Delta u|^{2}}{2}+|\Delta V|^{2}\right) d x \\
& =\left(\Delta V, \Delta\left[\nabla u V+V \nabla u^{T}+\omega_{12}(u)(V A-A V)\right.\right. \\
& \quad-\gamma(V A-A V)])+\left(\Delta u, \Delta \nabla \cdot\left(V V^{T}\right)\right)+(\Delta u, 2 \Delta \nabla \cdot V) \\
& \quad+(2 \Delta V, \Delta D(u))+\mu(\Delta u, \Delta \Delta u)-(\Delta u,[\Delta(u \cdot \nabla u)-u \cdot \nabla \Delta u]) \\
& \quad-(2 \Delta V,[\Delta(u \cdot \nabla V)-u \cdot \nabla \Delta V]) .
\end{aligned}
$$

Applying similar arguments as before, we conclude that

$$
\left\{\begin{array}{c}
\int_{R^{2}} u \cdot \nabla\left(\frac{|\Delta u|^{2}}{2}+|\Delta V|^{2}\right) d x+(\Delta u, \nabla \Delta p) \\
\quad-\mu(\Delta u, \Delta \Delta u)=\mu\|\Delta \nabla u\|^{2} \\
(\Delta u, 2 \Delta \nabla \cdot V)+(2 \Delta V, \Delta D(u))=0 .
\end{array}\right.
$$

To estimate the rest terms of the right side of (4.6), and also for later use, we need the following Lemmas. Their proofs can found in many literatures, see [14], for example.

Lemma 4.1. Assume $v \in W^{k, 2}\left(R^{2}\right), k \geq 3$. The following interpolation inequalities hold.

1. For $1 \leq s \leq k$,

$$
\begin{aligned}
& \|v\|_{L^{4}} \leq C\|v\|^{1-\frac{1}{2 s}}\left\|\nabla^{s} v\right\|^{\frac{1}{2 s}}, \\
& \|\nabla v\|_{L^{4}} \leq C\|v\|^{1-\frac{3}{2(s+1)}}\left\|\nabla^{s} \nabla v\right\|^{\frac{3}{2(s+1)}}, \\
& \|\Delta v\|_{L^{4}} \leq C\|v\|^{1-\frac{5}{2(s+2)}}\left\|\nabla^{s} \Delta v\right\|^{\frac{5}{2(s+2)}} \text {. }
\end{aligned}
$$

2. For $2 \leq s \leq k$,

$$
\begin{aligned}
\|v\|_{L^{\infty}} & \leq C\|v\|^{1-\frac{1}{s}}\left\|\nabla^{s} v\right\|^{\frac{1}{s}} \\
\|\nabla v\|_{L^{\infty}} & \leq C\|v\|^{1-\frac{2}{s+1}}\left\|\nabla^{s} \nabla v\right\|^{\frac{2}{s+1}} .
\end{aligned}
$$

The following Lemma will also be used for several times below.

Lemma 4.2. Assume $f, g \in H^{s}\left(R^{2}\right)$ for some $s>0$. Then there exists a universal constant $C$ depending only on s such that

$$
\left\|\nabla^{s}(f g)\right\| \leq C\left(\|f\|_{L^{\infty}}\left\|\nabla^{s} g\right\|+\|g\|_{L^{\infty}}\left\|\nabla^{s} f\right\|\right) .
$$


Now we estimate the second line of (4.6). Invoking the definition of $\gamma$ in (2.9), and with the aid of Lemma 4.2, we deduce that

$$
\begin{aligned}
& \left|\left(\Delta V, \Delta\left[\nabla u V+V \nabla u^{T}+\omega_{12}(u)(V A-A V)-\gamma(V A-A V)\right]\right)\right| \\
& \leq C\|\Delta V\|\left(\|\Delta V\|\|\nabla u\|_{L^{\infty}}+\|\nabla \Delta u\|\|V\|_{L^{\infty}}+\|\nabla \Delta u\|\|V\|_{L^{\infty}}^{2}\right. \\
& \left.\quad+\|\nabla u\|_{L^{\infty}}\left[\|V\|_{L^{\infty}}\|\Delta V\|+\|V\|_{L^{\infty}}^{2}\left\|\Delta \frac{1}{2+\operatorname{tr} V}\right\|\right]\right) \\
& \leq C \mu\|V\|_{H^{2}}\left(\|\nabla u\|^{2}+\|\nabla \Delta u\|^{2}+\frac{1}{\mu^{2}}\|\Delta V\|^{2}\right)
\end{aligned}
$$

provided that

$$
\|V\|_{L^{\infty}}<1
$$

which will be verified below.

Similarly, it is easy to show that

$$
\begin{aligned}
& \left|\left(\Delta u, \Delta \nabla \cdot\left(V V^{T}\right)\right)\right| \\
& \leq\|V\|_{L^{\infty}}\|\Delta V\|\|\nabla \Delta u\| \\
& \leq C \mu\|V\|_{H^{2}}\left(\|\nabla \Delta u\|^{2}+\frac{1}{\mu^{2}}\|\Delta V\|^{2}\right) .
\end{aligned}
$$

At last, it remains to estimate the last line of (4.6). Again, by Sobolev imbedding theorem and Lemma 4.2, it leads to

$$
\begin{aligned}
& -(\Delta u,[\Delta(u \cdot \nabla u)-u \cdot \nabla \Delta u])-(2 \Delta V,[\Delta(u \cdot \nabla V)-u \cdot \nabla \Delta V]) \mid \\
\leq & C\|\Delta u\|\left(\|\nabla u\|_{L^{\infty}}\|\Delta u\|+\|\nabla \nabla u\|\|u\|_{L^{\infty}}\right) \\
& +C\|\Delta V\|\left(\|\nabla u\|_{L^{\infty}}\|\Delta V\|+\|\nabla \nabla u\|\|V\|_{L^{\infty}}\right) \\
\leq & C(1+\mu)\left(\|u\|_{H^{2}}+\|V\|_{H^{2}}\right)\left(\|\nabla u\|^{2}+\|\nabla \Delta u\|^{2}+\frac{1}{\mu^{2}}\|\Delta V\|^{2}\right) .
\end{aligned}
$$

Combining all these estimates (4.7)-(4.11) with (4.5), and then noting (4.6), we can finally conclude that

$$
\begin{aligned}
& \frac{1}{2} \frac{d}{d t} \int_{R^{2}}\left(|u|^{2}+|\Delta u|^{2}+2|V|^{2}+2|\Delta V|^{2}\right) d x+\mu\left(\|\nabla u\|^{2}+\|\nabla \Delta u\|^{2}\right) \\
& \leq C(1+\mu)\left(\|u\|_{H^{2}}+\|V\|_{H^{2}}\right)\left(\|\nabla u\|^{2}+\|\nabla \Delta u\|^{2}+\frac{1}{\mu^{2}}\|\Delta V\|^{2}\right)
\end{aligned}
$$

provided (4.9) holds.

\section{Step 2. Weak Dissipation for The Strain Matrix $V$.}

To get dissipative energy estimates, by (4.12), it is clear that we should explore the dissipation of $V$. 
Let

$$
w=\Delta u+\frac{2}{\mu} \nabla \cdot V .
$$

Now we apply $\Delta$ to the second equation of system (1.2), and respectively, $\frac{1}{\mu} \nabla \cdot$ to the third one, and then adding up the outcomes to get a new equation. In terms of $w$ introduced in (4.13), this new equation can be written as

$$
w_{t}+u \cdot \nabla w+\nabla \Delta p=\mu \Delta w+\frac{1}{\mu} \Delta u+f .
$$

where

$$
\begin{aligned}
f & =-[\Delta(u \cdot \nabla u)-u \cdot \nabla \Delta u]-\frac{2}{\mu}[\nabla \cdot(u \cdot \nabla V) \\
& -u \cdot \nabla(\nabla \cdot V)]+\Delta \nabla \cdot\left(V V^{T}\right)+\frac{1}{\mu} \nabla \cdot\left[\left(\nabla u V+V \nabla u^{T}\right)\right. \\
& \left.+\omega_{12}(u)(V A-A V)-\gamma(V A-A V)\right] .
\end{aligned}
$$

Take the inner product of (4.14) with $w$ in $\left(L^{2}\left(R^{2}\right)\right)^{2}$, and use the similar arguments as in (4.2), we have

$$
\frac{1}{2} \frac{d}{d t} \int_{R^{2}}|w|^{2} d x+\mu\|\nabla w\|^{2}=(\Delta p, \nabla \cdot w)+\frac{1}{\mu}(\Delta u, w)+(f, w) .
$$

As before, we should estimate the right side of (4.16) term by term. Firstly, it is obvious that

$$
\frac{1}{\mu}|(\Delta u, w)| \leq \frac{\mu}{4}\|\nabla w\|^{2}+\frac{1}{\mu^{3}}\|\nabla u\|^{2} .
$$

On the other hand, noting that $\nabla \cdot u=0$, we can use integration by parts and Lemma 4.2 to estimate the last term of the right side of (4.16) as follows:

$$
\begin{aligned}
& |(f, w)| \leq\left|\left(\left[\Delta\left(u_{i} u_{j}\right)-u_{i} \Delta u_{j}\right], \nabla_{i} w_{j}\right)\right|+\left|\left(\Delta\left(V V^{T}\right), \nabla w\right)\right| \\
& \quad+\frac{1}{\mu} \mid\left(\left[\left(\nabla u V+V \nabla u^{T}\right)+\omega_{12}(u)(V A-A V)-\gamma(V A-A V)\right],\right. \\
& \quad \nabla w)\left|+\frac{2}{\mu}\right|\left(\left[\nabla_{k} \cdot\left(u_{i} V_{j k}\right)-u_{i}\left(\nabla_{k} V_{j k}\right)\right], \nabla_{i} w_{j}\right) \mid \\
& \leq C\|\nabla w\|\left[\left(\|u\|_{L^{\infty}}\|\Delta u\|+\|\nabla u\|_{L^{\infty}}\|\nabla u\|\right)\right. \\
& \left.\quad+\frac{1}{\mu}\|V\|_{L^{\infty}}\|\nabla u\|+\|V\|_{L^{\infty}}\|\Delta V\|+\frac{1}{\mu}\|V\|_{L^{\infty}}^{2}\|\nabla u\|\right] \\
& \leq C\left(\mu+\frac{1}{\mu}\right)\left(\|u\|_{H^{2}}+\|V\|_{H^{2}}\right) \\
& \quad \times\left(\|\nabla u\|^{2}+\|\Delta \nabla u\|^{2}+\|\nabla w\|^{2}+\frac{1}{\mu^{2}}\|\Delta V\|^{2}\right)
\end{aligned}
$$


provided $\|V\|_{L^{\infty}}<1$.

It also remains to estimate the first term of the right side of (4.16). For this purpose, we apply the divergence operator for the momentum equation of system (1.2) to get

$$
\Delta p=-\operatorname{tr}(\nabla u \nabla u)+\nabla \cdot\left[\nabla \cdot\left(V V^{T}\right)\right]+2 \nabla \cdot(\nabla \cdot V) .
$$

Thus, by (4.19), Lemma 4.1, Lemma 4.2 and Sobolev imbedding theorem, we can easily deduce that

$$
\begin{aligned}
& |(\Delta p, \nabla w)| \leq|(\operatorname{tr}(\nabla u \nabla u), \nabla w)| \\
& \quad+\left|\left(\nabla \cdot\left[\nabla \cdot\left(V V^{T}\right)\right], \nabla w\right)\right|+2|(\nabla \cdot(\nabla \cdot V), \nabla w)| \\
& \leq C \mid\|\nabla w\|\left(\|\nabla u\|_{L^{4}}^{2}+\|V\|_{L^{\infty}}\|\Delta V\|\right) \\
& \quad+\frac{\mu}{4}\|\nabla w\|^{2}+\frac{1}{\mu}\|\nabla \cdot(\nabla \cdot V)\|^{2} \\
& \leq C(1+\mu)\left(\|u\|_{H^{2}}+\|V\|_{H^{2}}\right)\left(\|\nabla u\|^{2}+\|\nabla \Delta u\|^{2}\right. \\
& \left.\quad+\|\nabla w\|^{2}+\frac{1}{\mu^{2}}\|\Delta V\|^{2}\right)+\frac{\mu}{4}\|\nabla w\|^{2}+\frac{1}{\mu}\|\nabla \cdot(\nabla \cdot V)\|^{2} .
\end{aligned}
$$

We must deal with the last term of the above estimates. Now Lemma 3.3 enters the argument in this stage. Noting the definition of $\nabla^{\perp}$, it is easy to see that $\nabla \cdot \nabla^{\perp}=0$. Thus, by Lemma 4.1 and a straightforward calculation, we have

$$
\begin{aligned}
& \frac{2}{\mu}\|\nabla \cdot(\nabla \cdot V)\|^{2}=\frac{2}{\mu}\|\nabla \cdot(A V \nabla \theta)\|^{2} \\
& \leq \frac{C}{\mu}\left(\|\nabla V\|_{L^{4}}\|\nabla \theta\|_{L^{4}}\right)^{2}+\left(\|V\|_{L^{\infty}}\left\|\nabla^{2} \theta\right\|\right)^{2} \\
& \leq \frac{C}{\mu}\|\nabla V\|\|\nabla \theta\|\|\Delta V\|\|\Delta \theta\|+\frac{C}{\mu}\|V\|_{L^{\infty}}^{2}\|\Delta \theta\|^{2} \\
& \leq C(1+\mu)\left(\|V\|_{H^{2}}+\|u\|_{H^{2}}\right)\left(\|\nabla \Delta u\|^{2}+\frac{1}{\mu^{2}}\|\Delta \theta\|^{2}+\frac{1}{\mu^{2}}\|\Delta V\|^{2}\right)
\end{aligned}
$$

provided (4.9) is satisfied and

$$
\|\nabla \theta\| \leq 1
$$

holds.

Finally, the combination of (4.16)-(4.21) leads to

$$
\begin{aligned}
& \frac{d}{d t} \int_{R^{2}}|w|^{2} d x+\mu\|\nabla w\|^{2} \\
& \leq C\left(\mu+\frac{1}{\mu}\right)\left(\|u\|_{H^{2}}+\|V\|_{H^{2}}\right)\left(\|\nabla u\|^{2}+\|\Delta \nabla u\|^{2}\right. \\
& \left.\quad+\|\nabla w\|^{2}+\frac{1}{\mu^{2}}\|\Delta V\|^{2}+\frac{1}{\mu^{2}}\|\Delta \theta\|^{2}\right)+\frac{2}{\mu^{3}}\|\nabla u\|^{2}
\end{aligned}
$$


provided (4.22) holds.

\section{Step 3. Weak Dissipation for $\nabla \theta$.}

At this stage, to extract the dissipative nature of the system, it is clear that we need also explore some kind of weak dissipation of $\Delta \theta$. For this purpose, we introduce the auxiliary variable $\Theta$ :

$$
\Theta=\Delta u+\frac{2}{\mu} \nabla^{\perp} \theta
$$

Rewrite the momentum equation as follows:

$$
u_{t}+u \cdot \nabla u+\nabla p=\mu \Delta u+2 \nabla^{\perp} \theta+\nabla \cdot\left(V V^{T}\right)+2 A V \nabla \theta .
$$

Then, apply $\Delta$ to (4.25) and $\frac{2}{\mu} \nabla \cdot$ to the last equation of (1.2), and then add up the resulting equations together, we obtain a new equation in terms of $\Theta$ :

$$
\Theta_{t}+u \cdot \nabla \Theta+\nabla \Delta p=\Delta \Theta+\frac{1}{\mu} \Delta u+g
$$

where

$$
\begin{aligned}
g & =\Delta \nabla \cdot\left(V V^{T}\right)+2 \Delta(A V \nabla \theta)+\frac{2}{\mu} \nabla^{\perp} \gamma \\
& -[\Delta(u \cdot \nabla u)-u \cdot \nabla \Delta u]-\frac{2}{\mu}\left(\begin{array}{c}
-\nabla_{2} u \cdot \nabla \theta \\
\nabla_{1} u \cdot \nabla \theta
\end{array}\right) .
\end{aligned}
$$

Similarly, we take the $L^{2}$ inner product of $(4.26)$ with $\Theta$ to get

$$
\frac{1}{2} \frac{d}{d t} \int_{R^{2}}|\Theta|^{2} d x+\mu\|\nabla \Theta\|^{2}=(\Delta p, \nabla \cdot \Theta)+\frac{1}{\mu}(\Delta u, \Theta)+(g, \Theta) .
$$

By the expression of $\Theta$ in (4.24) and the first equation of system (1.2), it is easy to see that

$$
(\Delta p, \nabla \cdot \Theta)=0
$$

A straightforward computation implies

$$
\frac{1}{\mu}|(\Delta u, \Theta)| \leq \frac{\mu}{4}\|\nabla \Theta\|^{2}+\frac{1}{\mu^{3}}\|\nabla u\|^{2} .
$$

To estimate the last term in (4.28), we insert (4.27) into $(g, \Theta)$ and then use integration by parts to yield

$$
\begin{aligned}
& |(g, \Theta)| \leq\left|\left(\left[\Delta\left(u_{i} u_{j}\right)-u_{i} \Delta u_{j}\right], \nabla_{i} \Theta_{j}\right)\right|+\frac{2}{\mu}\left|\left(\left(\begin{array}{c}
-\nabla_{2} \theta \nabla_{2} u \\
\nabla_{1} \theta \nabla_{1} u
\end{array}\right), \Theta\right)\right| \\
& \quad+\left|\left(\Delta\left(V V^{T}\right), \nabla \Theta\right)\right|+\frac{2}{\mu}\left|\left(\gamma, \nabla^{\perp} \cdot \Theta\right)\right|+2|(\Delta(A V \nabla \theta), \Theta)| .
\end{aligned}
$$


By a similar argument as before, we have

$$
\begin{aligned}
& \left|\left(\left[\Delta\left(u_{i} u_{j}\right)-u_{i} \Delta u_{j}\right], \nabla_{i} \Theta_{j}\right)\right|+\left|\left(\Delta\left(V V^{T}\right), \nabla \Theta\right)\right|+2|(\Delta(A V \nabla \theta), \Theta)|(4) \\
& \leq C\|\nabla \Theta\|\left[\left(\|u\|_{L^{\infty}}\|\Delta u\|+\|\nabla u\|_{L^{\infty}}\|\nabla u\|\right)+\|V\|_{L^{\infty}}\|\Delta V\|\right] \\
& \quad+C\|\nabla \Theta\|\left(\|V\|_{L^{\infty}}\|\Delta \theta\|+\|\nabla V\|_{L^{4}}\|\nabla \theta\|_{L^{4}}\right) \\
& \leq C\left(1+\mu^{\frac{3}{2}}\right)\left(\|u\|_{H^{2}}+\|V\|_{H^{2}}+\frac{1}{\mu}\|\nabla \theta\|\right) \\
& \quad \times\left(\|\nabla u\|^{2}+\|\Delta \nabla u\|^{2}+\|\nabla \Theta\|^{2}+\frac{1}{\mu^{2}}\|\Delta V\|^{2}+\frac{1}{\mu^{2}}\|\Delta \theta\|^{2}\right)
\end{aligned}
$$

where we used Lemma 4.1 and Lemma 4.2. On the other hand, by (2.9), it follows that

$$
\begin{aligned}
& \frac{2}{\mu}\left|\left(\left(\begin{array}{c}
-\nabla_{2} \theta \nabla_{2} u \\
\nabla_{1} \theta \nabla_{1} u
\end{array}\right), \Theta\right)\right|+\frac{2}{\mu}\left|\left(\gamma, \nabla^{\perp} \cdot \Theta\right)\right| \\
& \leq \frac{C}{\mu}\left(\|\nabla u\|\|\nabla \theta\|_{L^{4}}\|\Theta\|_{L^{4}}+\|V\|_{L^{\infty}}\|\nabla u\| \nabla \Theta \|\right) \\
& \leq \frac{C}{\mu}\left(\|\nabla u\|\|\nabla \theta\|^{\frac{1}{2}}\|\Theta\|^{\frac{1}{2}}\|\Delta \theta\|^{\frac{1}{2}}\|\nabla \Theta\|^{\frac{1}{2}}+\|V\|_{L^{\infty}}\|\nabla u\| \nabla \Theta \|\right) \\
& \leq C\left(1+\frac{1}{\mu}\right)\left(\|\Theta\|+\|V\|_{H^{2}}+\frac{1}{\mu}\|\nabla \theta\|\right) \\
& \quad \times\left(\|\nabla u\|^{2}+\|\nabla \Theta\|^{2}+\frac{1}{\mu^{2}}\|\Delta \theta\|^{2}\right) .
\end{aligned}
$$

The combination of (4.31)-(4.33) implies that

$$
\begin{aligned}
& |(g, \Theta)| \leq C\left(\mu^{2}+\frac{1}{\mu}\right)\left(\|u\|_{H^{2}}+\|V\|_{H^{2}}+\|\Theta\|+\frac{1}{\mu}\|\nabla \theta\|\right) \\
& \quad \times\left(\|\nabla u\|^{2}+\|\Delta \nabla u\|^{2}+\|\nabla \Theta\|^{2}+\frac{1}{\mu^{2}}\|\Delta \theta\|^{2}+\frac{1}{\mu^{2}}\|\Delta V\|^{2}\right) .
\end{aligned}
$$

Finally, combining (4.28)-(4.30) with (4.34), and noting the definition of $\Theta$ in (4.24), we arrive at

$$
\begin{aligned}
& \frac{d}{d t} \int_{R^{2}}|\Theta|^{2} d x+\mu\|\nabla \Theta\|^{2} \\
& \leq \frac{C}{\mu^{3}}\|\nabla u\|^{2}+C\left(\mu^{2}+\frac{1}{\mu}\right)\left(\|u\|_{H^{2}}+\|V\|_{H^{2}}+\|\Theta\|\right) \\
& \quad \times\left(\|\nabla u\|^{2}+\|\Delta \nabla u\|^{2}+\|\nabla \Theta\|^{2}+\frac{1}{\mu^{2}}\|\Delta V\|^{2}\right) .
\end{aligned}
$$

Step 4. A Priori Weakly Dissipative Energy Estimates. 
This step ties everything together. Firstly, by Hodge's decomposition, we calculate

$$
\begin{aligned}
& \Delta V=\nabla \nabla \cdot V-\nabla \times \nabla \times V \\
& =\nabla \nabla \cdot V-\left(\begin{array}{ll}
\nabla_{2}\left(\nabla_{1} V_{12}-\nabla_{2} V_{11}\right) & -\nabla_{1}\left(\nabla_{1} V_{12}-\nabla_{2} V_{11}\right) \\
\nabla_{2}\left(\nabla_{1} V_{22}-\nabla_{2} V_{21}\right) & -\nabla_{1}\left(\nabla_{1} V_{22}-\nabla_{2} V_{21}\right)
\end{array}\right) \\
& =\nabla \nabla \cdot V+\left(\nabla^{\perp}\right)^{2} t r V-\nabla^{\perp}(A \nabla \cdot V) .
\end{aligned}
$$

Thus, noting (3.2), and by (4.13) and Lemma 4.2, we obtain

$$
\begin{aligned}
& \|\Delta V\| \leq C \mu(\|\Delta \nabla u\|+\|\nabla w\|+\|\Delta \operatorname{tr} V\|) \\
& \leq C \mu(\|\Delta \nabla u\|+\|\nabla w\|)+C \mu\|\Delta V\|\|V\|_{L^{\infty}}
\end{aligned}
$$

which implies that

$$
\|\Delta V\| \leq C \mu(\|\Delta \nabla u\|+\|\nabla w\|)
$$

provided that

$$
\|V\|_{L^{\infty}} \leq \frac{1}{2 \mu C} .
$$

On the other hand, by (4.24),

$$
\left\{\begin{array}{l}
\|\nabla \theta\| \leq C \mu(\|\Delta u\|+\|\Theta\|), \\
\|\Delta \theta\| \leq C \mu(\|\Delta \nabla u\|+\|\nabla \Theta\|) .
\end{array}\right.
$$

Now we can proceed with the dissipative energy method. Plug (4.39) into (4.12), we find that

$$
\begin{aligned}
& \frac{1}{2} \frac{d}{d t} \int_{R^{2}}\left(|u|^{2}+|\Delta u|^{2}+2|V|^{2}+2|\Delta V|^{2}\right) d x+\mu\left(\|\nabla u\|^{2}+\|\nabla \Delta u\|^{2}\right) \\
& \leq C(1+\mu)\left(\|u\|_{H^{2}}+\|V\|_{H^{2}}\right)\left(\|\nabla u\|^{2}+\|\nabla \Delta u\|^{2}+\|\nabla w\|^{2}\right) .
\end{aligned}
$$

Similarly, insert (4.37) and (4.39) into (4.23) and (4.35), and then add the resulting inequalities together, we obtain that

$$
\begin{aligned}
& \frac{d}{d t} \int_{R^{2}}\left(|w|^{2}+|\Theta|^{2}\right) d x+\mu\left(\|\nabla w\|^{2}\|+\nabla \Theta\|^{2}\right) \\
& \leq C\left(\mu^{2}+\frac{1}{\mu}\right)\left(\|u\|_{H^{2}}+\|V\|_{H^{2}}+\|\Theta\|\right)\left(\|\nabla u\|^{2}+\|\Delta \nabla u\|^{2}\right. \\
& \left.\quad+\|\nabla w\|^{2}+\|\nabla \Theta\|^{2}\right)+\frac{C}{\mu^{3}}\|\nabla u\|^{2} .
\end{aligned}
$$

Combining (4.40) with (4.41), we finally arrive at

$$
\begin{aligned}
& \frac{d}{d t} \int_{R^{2}}\left[\left(|u|^{2}+|\Delta u|^{2}+2|V|^{2}+2|\Delta V|^{2}\right)+|w|^{2}+|\Theta|^{2}\right] d x \\
& \quad+\mu\left(\|\nabla u\|^{2}+\|\nabla \Delta u\|^{2}+\|\nabla w\|^{2}+\|\nabla \Theta\|^{2}\right) \\
& \leq \frac{C}{\mu^{3}}\|\nabla u\|^{2}+C\left(\mu^{2}+\frac{1}{\mu}\right)\left(\|u\|_{H^{2}}+\|V\|_{H^{2}}+\|\Theta\|\right) \\
& \quad \times\left(\|\nabla u\|^{2}+\|\nabla \Delta u\|^{2}+\|\nabla w\|^{2}+\|\nabla \Theta\|^{2}\right) .
\end{aligned}
$$


Therefore, if the initial data is sufficiently small, we can find some positive time $T$, such that

$$
\|u\|_{H^{2}}+\|V\|_{H^{2}}+\|\Theta\| \leq \frac{\mu^{2}}{2 C\left(1+\mu^{3}\right)}
$$

for all $0 \leq t \leq T$. Moreover, in this case, (4.42) implies that

$$
\begin{aligned}
& \frac{d}{d t} \int_{R^{2}}\left[\left(|u|^{2}+|\Delta u|^{2}+2|V|^{2}+2|\Delta V|^{2}\right)+|w|^{2}+|\Theta|^{2}\right] d x \\
& \quad+\mu\left(\|\nabla u\|^{2}+\|\nabla \Delta u\|^{2}+\|\nabla w\|^{2}+\|\nabla \Theta\|^{2}\right) \leq \frac{C}{\mu^{3}}\|\nabla u\|^{2} .
\end{aligned}
$$

Thus, noting (4.13), (4.24), and by integrating the above inequality from 0 to $t, 0 \leq$ $t \leq T$, with respect to $t$, we obtain

$$
\begin{aligned}
& \left(\|u\|_{H^{2}}^{2}+\|V\|_{H^{2}}^{2}+\|w\|^{2}+\|\Theta\|^{2}\right) \\
& \quad+\mu \int_{0}^{\infty}\left(\|\nabla u\|_{H^{2}}^{2}+\|\nabla w\|^{2}+\|\nabla \Theta\|^{2}\right) d t \\
& \leq C\left(\left\|u_{0}\right\|_{H^{2}}^{2}+\left\|V_{0}\right\|_{H^{2}}^{2}+\left\|w_{0}\right\|^{2}+\left\|\Theta_{0}\right\|^{2}\right)+\frac{C}{\mu^{3}} \int_{0}^{\infty}\|\nabla u\|^{2} d t \\
& \leq C\left(1+\frac{1}{\mu^{4}}\right)\left(\left\|u_{0}\right\|_{H^{2}}^{2}+\left\|V_{0}\right\|_{H^{2}}^{2}+\left\|\nabla \theta_{0}\right\|_{H^{1}}^{2}\right),
\end{aligned}
$$

where in the last inequality, we used the basic energy law (4.4). Then, it follows from (4.44) that if the initial data satisfies

$$
\left\|u_{0}\right\|_{H^{2}}^{2}+\left\|V_{0}\right\|_{H^{2}}^{2}+\|\nabla \theta\|_{H^{1}}^{2} \leq \frac{\mu^{8}}{M\left(1+\mu^{10}\right)}
$$

for a big enough constant $M\left(M \geq 2 C^{3}\right)$ independent of $\mu$, then (4.43) will still hold for all the latter time $t \geq T$ with a uniform constant $C$ independent of $t$ and $\mu$. Furthermore, when (4.45) holds, we can deduce from (4.44) that

$$
\begin{aligned}
& \left(\|u\|_{H^{2}}^{2}+\|V\|_{H^{2}}^{2}+\|w\|^{2}+\|\Theta\|^{2}\right) \\
& \quad+\mu \int_{0}^{\infty}\left(\|\nabla u\|_{H^{2}}^{2}+\|\nabla w\|^{2}+\|\nabla \Theta\|^{2}\right) d t \\
& \leq C\left(\left\|u_{0}\right\|_{H^{2}}^{2}+\left\|V_{0}\right\|_{H^{2}}^{2}+\left\|w_{0}\right\|^{2}+\left\|\Theta_{0}\right\|^{2}\right)+\frac{C}{\mu^{3}} \int_{0}^{\infty}\|\nabla u\|^{2} d t \\
& \leq \frac{\mu^{4}\left(1+\mu^{4}\right)}{C^{2}\left(1+\mu^{10}\right)}
\end{aligned}
$$

provided $C$ is big enough.

At last, we should show (4.9), (4.22) and (4.38). By (4.43) and Sobolev imbedding theorem, we have

$$
\|V\|_{L^{\infty}} \leq c\|V\|_{H^{2}} \leq \frac{c \mu^{2}}{2 C\left(\mu^{3}+1\right)}
$$


for appropriate constants $c$ and $C$. Thus, we have

$$
\|V\|_{L^{\infty}} \leq \begin{cases}\frac{1}{\mu} \leq 1, & \text { for } \quad \mu \geq 1 \\ 1 \leq \frac{1}{\mu}, & \text { for } \quad \mu \leq 1\end{cases}
$$

which means that (4.9) and (4.38) hold. To show (4.22), we deduce from (4.43) and (4.24) that

$$
\|\nabla \theta\| \leq 2 \mu\left(\|\Theta\|+\|u\|_{H^{2}}\right) \leq \frac{\mu^{3}}{C\left(1+\mu^{3}\right)} \leq 1 .
$$

The proof of Theorem 2.2 is completed.

\section{Acknowledgments}

The author would like to thank professor Chun Liu and professor Yi Zhou for their constructive suggestions and helpful discussions. The author was partially supported by the NSFC grant 10801029, the PSFC grants 20070410160 and 200801175.

\section{References}

[1] R. Agemi, Global existence of nonlinear elastic waves, Invent. Math., 142(2) (2000), 225-250.

[2] J. Y. Chemin and N. Masmoudi, About lifespan of regular solutions of equations related to viscoelastic fluids, SIAM J. Math. Anal., 33(1) (2001), 84-112.

[3] Y. Chen and P. Zhang, The global existence of small solutions to the incompressible viscoelastic fluid system in 2 and 3 space dimensions. Comm. Partial Differential Equations 31 (2006), no. 10-12, 1793-1810.

[4] D. Christodoulou, Global existence of nonlinear hyperbolic equations for small data, Comm. Pure. Appl. Math., 39 (1986), 267-286.

[5] K. O. Friedrichs, On the boundary-value problems of the theory of Elasticity and Korn's inequality, Ann. Math., 48 (1947), 441-471.

[6] F. John, Rotation and strain, Comm. Pure Appl. Math., 14 (1961), 391-413.

[7] F. John, Distance changes in deformations with small strain. 1970 Studies and Essays (Presented to Yu-why Chen on his 60th Birthday, April 1, 1970) pp. 1-15 Math. Res. Center, Nat. Taiwan Univ., Taipei

[8] F. John and S. Klainerman, Almost global existence to nonlinear wave equations in three space dimensions, Comm. Pure Appl. Math., 37 (1984), 443-455. 
[9] P. Kessenich, Global existence with small initial data for three-dimensional incompressible isotropic viscoelastic materials, preprint. available at http://arxiv.org/abs/0903.2824v1

[10] S. Klainerman, The null condition and global existence to nonlinear wave equations, Lect. in Appl. Math., 23 (1986), 293-326.

[11] S. Klainerman and T. C. Sideris, On almost global existence for nonrelativistic wave equations in 3D, Comm. Pure Appl. Math. 49 (1996), 307-322.

[12] Z. Lei, Global existence of classical solutions for some Oldroyd-B model via the incompressible limit, Chin. Ann. Math. Ser. B, 27 (2006), no. 5, 565-580.

[13] Z. Lei, C. Liu and Y. Zhou, Global existence for a 2D incompressible viscoelastic model with small strain. Comm. Math. Sci., 5 (2007), no. 3, 595-616.

[14] Z. Lei, C. Liu and Y. Zhou, Global solutions for incompressible viscoelastic fluids. Arch. Ration. Mech. Anal., 188 (2008), no. 3, 371-398.

[15] Z. Lei and Y. Zhou, Global existence of classical solutions for 2D Oldroyd model via the incompressible limit, SIAM J. Math. Anal., 37 (2005), no. 3, 797-814.

[16] F. H. Lin and C. Liu, Nonparabolic dissipative systems modelling the flow of liquid crystals, Comm. Pure Appl. Math., 48(5) (1995), 501-537.

[17] F. H. Lin, C. Liu and P. Zhang, On hydrodynamics of viscoelastic fluids, Comm. Pure Appl. Math., 58(11) (2005), 1437-1471.

[18] P. L. Lions and N. Masmoudi, Global solutions for some Oldroyd models of nonNewtonian flows, Chinese Ann. Math. Ser. B 21(1) (2000), 131-146.

[19] C. Liu and N. J. Walkington, An Eulerian description of fluids containing viscohyperelastic particles, Arch. Rat. Mech Ana. 159 (2001), 229-252.

[20] T. C. Sideris, Nonresonance and global existence of prestressed nonlinear elastic waves, Ann. of Math., 151 (2000), 849-874.

[21] T. C. Sideris and B. Thomases, Global existence for three-dimensional incompressible isotropic elastodynamics via the incompressible limit. Comm. Pure Appl. Math. 58 (2005), no. 6, 750-788.

[22] T. C. Sideris and B. Thomases, Local energy decay for solutions of multidimensional isotropic symmetric hyperbolic systems. J. Hyperbolic Differ. Equ. 3 (2006), no. 4, 673-690.

[23] T. C. Sideris and B. Thomases, Global existence for three-dimensional incompressible isotropic elastodynamics. Comm. Pure Appl. Math. 60 (2007), no. 12, $1707-1730$. 